\begin{abstract}
Discreet Synthesizer
Allowing new ambient music to emerge through composition on semi-modular synths
\end{abstract}

By

Sonya Waters

\begin{abstract}
An exegesis
submitted to Victoria University of Wellington

in partial fulfilment of the requirements for the degree of Master of Musical Arts in Composition.
\end{abstract}

Victoria University of Wellington 2021 
Copyright by Sonya Waters All rights reserved May 2021 


\begin{abstract}
This exegesis is an exploration of ambient music through defining the music and examining the intentions, creative processes and listening objectives of ambient composers. The musical concepts and philosophy surrounding ambient music were introduced through the work and ideas of Brian Eno in the 1970s. His theories have been threaded throughout this exegesis and indeed throughout my own work. Eno's main beliefs about ambient music are that it should be gentle, unintrusive and as ignorable as it is interesting, a music that tints the environment where it is heard. Eno's ambient music uses a steady state texture, an atmosphere, and the music is partially or fully composed using chance and algorithmic processes. There are no dramatic events or harmonic changes and textural features which are normally in the background are bought to the foreground in ambient music. This is one of the fascinating differences between ambient music and other musical genres. I will examine these key features later in the exegesis.
\end{abstract}

Other artists who influenced the ambient genre will also be discussed. These wellknown and very important experimental composers were all searching for a new way to create music. Composers who use alternative methods for creating music that I found particularly interesting are Luigi Russolo, Pierre Schaffer, Claude Debussy, Erik Satie, John Cage, La Mont Young, Pauline Oliveros and Terry Riley. Through researching these composers, I have noticed a change in my own composition ideas and methods while making ambient music on semi-modular synthesizers. 


\section{List of Acknowledgments}

I would like to thank my supervisor Dugal McKinnon for his consistent guidance and encouraging feedback throughout the process of creating this exegesis and portfolio of ambient music. Dugal, thank you for recognising I am an ambient artist even before I realised it myself. Your suggestions for improving my compositions were appreciated and gave me much food for thought. Also, I could not have written this exegesis without your help in navigating such a difficult, but incredibly interesting subject area. Thank you so much!

Thanks also to Kim Canady for offering to help.

A big thank you to my partner Ben Howe for always being supportive and encouraging - and for buying me a Moog synthesizer! 


\section{Discreet Synthesizer \\ Allowing new ambient music to emerge through composition on semi-modular synths}

\section{Introduction}

In this exegesis I will discuss the key elements and materials that define ambient music. I will also explore creative processes and listening objectives of ambient composers with particular focus on minimalism, drone music, looping and the use of environmental sound. I will reflect on my own composition methods and goals while making ambient music on semi-modular synthesizers and discuss how technology, and other composers have influenced my work, thoughts and ideas. The discussion of my work will be threaded through the exegesis, rather than discussed in an independent section. Similarly, technology will be tied into various parts of this exegesis, given that synthesis underpins much of the work I will be discussing, including my own.

Ambient music has been described as a musical genre that focusses primarily on timbre and atmosphere which distinguishes it from most other traditional forms of music where harmony, melody, and metric rhythm are defining central structures. Ambient could include music which has no obvious beat elements, uses synthesized sounds, natural soundscapes, or the tones of heavily reverberated acoustic instruments (Bates 2019). Wikipedia describes ambient music as evoking an atmosphere of calm and contemplation with no structured melody. The use of textural layers of sound which can be used to induce sleep, accompany yoga, meditation, aid study and relaxation are also associated with ambient. The genre is said to evoke an "atmospheric", "visual", or "unobtrusive quality.

https://en.wikipedia.org/wiki/Ambient_music (Wikipedia, Ambient Music) ${ }^{1}$ The Merriam-Webster's New Twentieth Century Dictionary defined ambient as "surrounding; encompassing on all sides; as, the ambient air" (Merriam-Webster, "Ambient"). The Sage International Encyclopedia of Music states ambient music is the only genre defined by spatial and background features (Bates 2019). Sound engineers have their own understanding of the word ambience. They perceive it as a spatial dimension relating to a degree of echo, delay or reverberation that is added to the music artificially using recording techniques (Tamm 1989). ${ }^{2}$

\footnotetext{
${ }^{1}$ https://en.wikipedia.org/wiki/Ambient_music. https://www.merriam-webster.com/dictionary/ambient https://en.wikipedia.org/wiki/Ambient_music.
} 
Brian Eno's ambient series are classic examples embodying all of the early core ambient elements of quietness, gentleness, a continual single atmosphere, nondevelopmental forms, repeating cyclic events, modal pitch sets, layered textures and an emphasis on timbre. The series is Ambient 1: Music for Airports (1978), Ambient 2: The Plateaux of Mirror (1980), Ambient 3: Day of Radiance (1981), Ambient 4: On Land (1982). Brian Eno's music has been described as “amorphous, open-ended, unstructured time, with undercurrents of foreboding, pockets of boredom and fleeting interludes of peace or reassessment" (Pareles 2020). A good example of ambient music is Robert Fripp \& Brian Eno's album Evening Star (1975). Track one, Wind on Water consists of effervescent sequences of synthesizer notes and layers of heavily reverbed guitar. The music bubbles and pulsates slowly, creating interesting rhythmic phasing effects and develops through play with different timbres and repeating patterns. As a whole the album is characterised by a gentle contemplative atmosphere, synthesizer sounds, reverbed guitar improvisations, and no drumkit elements.

Identifying the musical and conceptual elements that are common to ambient makes it possible to loosely define the genre. The essential elements are: a concern for timbre (or "tone colour"), atmospheres, vertical time through longform compositions, a steady state texture and generative techniques. Part of this discussion will be working through what these terms mean and how they help define ambient music. Alongside these musical elements ambient artists have adopted new musical technologies to create and explore their work. Technology is a theme woven through the following sections.

Ambient music brings background timbres, textures and atmospheres to the fore using a variety of heavily effected materials such as drones, loops, and environmental sounds. There is no obvious emotional message or musical statement - the listener may interpret the meaning of the pieces in their own way. Ambient music can be music for focussed listening or used as background music. Leading ambient composer Brian Eno creates unobtrusive and emotionally neutral music he describes as "ignoresting" - switching between ignorable and interesting (Eno, liner notes Music for Airports (1978)). This statement allows listeners to drift back and forth between focussed listening and allowing the music to blend into the ambience of the environment. When creating music Eno uses a single pervasive atmosphere. He defines ambience "as atmosphere, or a surrounding influence: a tint" (Tamm 1989).

Vertical time has played an integral part in ambient music, not only in the actual length of pieces (which tend to be long) but also in the way time is perceived to exist. Vertical time is a term used to describe music that uses colouration and texture as its main focus instead of developing melodies, harmonies and rhythmic 
motifs (Tamm 1989). As ambient music focusses on timbre and tone colour, instead of more traditional musical traits, the listener explores sonic space without a point of reference giving the impression of timelessness. Pieces of longer duration allow the listener time to delve deeply into the central features of the music. One interesting ambient piece is Max Richter's Sleep Music (2015). Although Richter is not strictly an ambient composer his 8-hour continuous and uninterrupted Sleep Music album has been described as a slow-motion electronics and chamber ensemble hybrid (Haver Curran 2015). In his own words Richter (2015) described it as "an invitation to dream".

The composers and the music I am exploring in this exegesis has come about as a reaction against status quo musical practices in various eras from Luigi Russolo's intonarumori and his Art of Noises Manifesto (1913) to Brian Eno's Music for Airports (1978). Of particular interest are composers who were searching for new forms of musical expression and who were interested in challenging accepted norms to create something novel where there were few if any established rules or set pathways for creative success. In particular I will discuss how ambient composers subvert expectations by avoiding the use of common musical compositional elements such as harmonic and rhythmic developments. I will examine compositional methods allowing a degree of 'chance' in the creative process and investigate how the resulting materials have been created or assimilated into musical works. These ideas differ from traditional methods used by a composer working with pen and paper or even a DAW. The idea of the composer as listener is particularly important to the exegesis. Speaking generally, once new ideas have surfaced through chance processes, creative decisions are made about how these ideas will be organised and this involves an increased degree of listening, followed by curation of sounds, and the arranging and production of the music. There is an enormous range of ambient influenced music and every aspect of it cannot be covered here. Other significant areas that will be discussed are the concept of atmospheres, aural architecture, auditory spatial awareness, natural resonating environments, environmental sound and generative music techniques (which are closely related to the musical use of chance).

Artists from different countries around the world have their own spin on ambient music. German experimental band Tangerine Dream emerged as part of the Krautrock movement in the late 1960s. They made synthesized instrumental, longform music involving layers of texturized synthesizer drones using steady state methodology. Their album Atem (1973) was an ambient place of the imagination and futurism, exploring their fascination with space travel. The music was created using state of the art electronic equipment, but it was often perceived as cold and neutral, bringing to mind the philosophy of Schopenhauer or Spinoza - spacious, monumental, and detached from human emotion (Stubbs 2014). Over time other 
Krautrock artists besides Tangerine Dream moved into the ambient zone. Examples are Hans-Joachim Roedelius and Dieter Moebius from bands Cluster and Harmonia, and Holger Czukay from Can, who all went onto collaborate with Brian Eno creating the album Cluster and Eno (1977). Could Roedelius, Moebius and Czukay have ventured into ambient music as they continued to explore different manifestations of sound, after the Krautrock movement and their associated collaborations in bands had ended?

Reflection \#1 - My own interest in ambient music emerged as a reaction against the complexities and intensity of a Krautrock-inspired band I had been part of. After comparing Krautrock music from the 1970s with the music we were making in the band I wanted to produce material that was more sparce and minimal but also hypnotic and atmospheric. I started to explore a range of complementary sounds within a minimal palette of notes with the intention of moving away from song structure, drums and guitar music. I also wanted to allow ideas to emerge that were perhaps not part of the initial plan. Later in the studio production phase I could listen and examine the material and make decisions about what to include or exclude.

\section{Precursors to Ambient Music}

Historically ambient music was presented to the music listening public as a new musical genre in the 1970s through the work of Brian Eno. Eno's work is of course particularly significant and I will discuss it in detail in the next section - Defining Ambient Music. Some of the other composers recognised as presaging the ambient genre include Claude Debussy, Erik Satie, and John Cage. However, music that bears relationship to ambient can be traced at least as far back as the Gregorian chant of the Medieval period. In the $13^{\text {th }}$ century isorhythmic patterning can be seen as an example of the 'steady state texture' (Cummings 2019) which is at the core of ambient music and which I will discuss later in the exegesis. An isorhythm is a medieval compositional technique of continual repeating melodic patterns which are layered over other repeating vocal patterns. These are sung and the voice is used in an instrumental way with a free-flowing melody sung within a narrow range of notes. The rhythmic and melodic components are continually repeated asynchronously at different durations thereby creating constantly changing variations which will not repeat for a very long time. There is no set beginning or ending (Cummings 2019).

The work of Claude Debussy can be heard as influencing the ambient genre through his concept of vaguely suggesting something without defining it concretely. For this reason, Debussy was known as the first Impressionist composer (c. 1894- 
1915) exploring a sound world through intuition, unusual tonalities and the use of improvisation during the composition process (Prendergast 2003). Debussy chose methods of alluding to, while also blurring, the tonality underpinning the Western classical tradition. Debussy often used ambiguous non-harmonic structures involving unusual chords such as the dominant $13^{\text {th }}$, with much use of harmonic planing (parallel motion) across a complex vertical framework (Cope 1971). His impressionist piano pieces frequently used unresolved chords and avoid traditional harmony and counterpoint, as he traversed a world of sound beyond the rules and conventions of his era. Indeed, Debussy's orchestral masterpiece Prelude z l'apresmidi d'un faune (1884) was considered too unusual for audiences of the era (Prenderghast 2003). Debussy was searching for a new musical world and did so by exploring alternative scales, modal melodies, and adopting a floating liquidity in part inspired by Javanese gamelan music.

Eric Satie, a contemporary of Debussy, first approached what we now call ambient in 1917 when conceiving his "Furniture Music." Satie was aligned with the European Dada movement, a radical early-twentieth-century art movement challenging the grandiose bourgeois assumptions of nineteenth-century culture. Satie reacted against the pompous and ostentatious style of Wagner in particular when he produced his spare, unassuming, playful, and subversive music. The concept of unimposing background music - which is one of the ways that ambient music is defined - was not fully explored until decades later (Stubbs 2018). As Satie (1917) conceived it, Furniture Music is:

a music which is like furniture - a music, that is, which will be part of the noises of the environment.... softening the noises of the knives and forks, not dominating them, not imposing itself. It would fill up those heavy silences that sometimes fall between friends dining together. It would spare them the trouble of paying attention to their own banal remarks

Another composer who influenced the ambient genre through the use of environmental sound and chance music is American experimental composer John Cage, who began exploring aleatoric/chance composition methods in the 1950s as a way to free himself from individual musical taste and the traditions of music. Cage created a process for composing music by using the I Ching, an ancient Chinese symbol system used to identify order in chance events. Cage's process involved an elaborate system of throwing the I Ching coins to answer a series of questions. He wrote music according to the "answers" the coins prescribed (Auner 2013). Cage's music could be shocking and challenging. He advocated the use of any sound from the environment as a resource for making music and was interested in the way sound behaved outside of composed music. Indeed, Cage is most wellknown for his silent piece of music 4'33 (1952) which deliberately drew audiences' 
attention to all sound, including both musical and environmental sound. $\mathrm{He}$ described ambient as the sounds of the environment where one finds oneself (Schwarz 1996), an understanding which is closely linked to the features of ambient music I will explore in a later section. Cage's (1991) interest in ambient sound is best described in his own words:

When I hear what we call music, it seems to me that someone is talking. And talking about his feelings, or about his ideas of relationships. But when I hear traffic, the sound of traffic-here on Sixth Avenue, for instance-I don't have the feeling that anyone is talking. I have the feeling that sound is acting. And I love the activity of sound ... I don't need sound to talk to me.

\section{Related Research}

Other rather fascinating topics which link to ambient music are the ideas around how architectural and natural spaces effect our psyche and how we interpret our environment through sound. These are important because ambient music is often understood as being like an environment through its immersive and 'surrounding' qualities.

Aural architecture refers to the properties and qualities of a space that can be experienced by listening. Aural architecture is rarely considered when architects design a building. Auditory spatial awareness has a lower priority than visual attributes. The results of neglecting the auditory environment can be the creation of unintended physical effects such as echoes bouncing off walls in unsettling ways and enhancement of frequencies that are indirectly related to perception. While this could make interesting material for ambient composers, these perceptions are interpreted in unexpected ways by the brain and can influence our social behaviour. For example, a restaurant with many reflective surfaces creating echoes can make dining an unpleasant experience, or an empty train station could be interpreted as lonely and forbidding while an empty chapel is understood as a place of privacy and quiet contemplation (Blesser 2007). This links with Erik Satie's idea discussed above, of a music that is unimposing and part of the noises of the environment. Brian Eno also focussed his music on tinting the environment instead of dominating it.

Aural architecture, with its emphasis on the experience of acoustics, suggests that humans have understood and made use of ambience and resonance throughout history from prehistoric times to current day. This begs the question: has ambient music always existed and is it even possible to pin-point a set beginning for ambient as a specific form of sonic experience? Indeed, throughout history there is evidence 
humans have used and understood ambience. In the $9^{\text {th }}$ and $10^{\text {th }}$ centuries Medieval Catholic monks in Western and Central Europe were using the resonance of architectural spaces through Gregorian chant. The chants had a monophonic texture and were sung unaccompanied. Chants were performed in large reverberating churches and the slow layered qualities of the music were enhanced by the resonant features of the spaces. In this way, Gregorian chant used the aural architecture of internal reverbed chambers to convey a sense of awe and reverence. Naturally occurring reverb influenced the experience of sacred moments in those settings (Blesser 2007).

Even earlier in Palaeolithic times, there is evidence of humans possessing an enhanced ability for auditory spatial awareness. It is possible prehistoric humans understood how to use sound resonance in sacred cave spaces. Palaeolithic cave paintings have been found to be the most dense where the sound of the cave is the most resonant. There are a number of ideas suggesting the reasons for this. One is that resonant areas provide a way to navigate using the human voice as a sonar to manoeuvre through difficult and unpredictable dark cave pathways. As the sounds of the environment, animals, the human body, and echoes inside a cave preceded the development of language, it is likely early humans were highly aware of sound, experiencing and interpreting it through the body (Reznikoff 2008). Again, this ties into the environment, immersive qualities of ambient music. Indeed, Reznikoff suggests that strong aural resonances connect to our deepest perceptions of sound; that of hearing sounds inside the womb before interpreting speech and visual cues. In the womb our most primitive perceptions are developing and experienced in a corporeal way. External sounds are differentiated from the sounds of our own heart beat and the internal sounds of our mother's body. On this sense our first experience is ambient. 


\section{Defining Ambient Music}

\section{Timbre, Deep Listening, The Steady State, Atmospheres, and Vertical Time}

As described in the previous section ambient music focusses on bringing background features into the foreground through a focus on timbre, the use of a single atmosphere, and vertical time through longform compositions where the listener explores textural qualities over a longer period of time. Other important elements are the use of a steady state texture and generative techniques. Brian Eno describes ambient music as tinting the environment, and as a music that had been created by a degree of chance using generative processes.

By looking at timbre, vertical time, the steady state, atmospheres, and generative processes as the central elements of ambient music it is possible to understand what makes this music interesting, challenging and vastly different from other music genres.

\section{Timbre}

Timbre considered as "tone colour", is the quality of sound that distinguishes one instrument from another. Timbre has become a central musical concern for many musicians and composers in recent decades although this is not to forget the longestablished practice of orchestration; how composers use and combine the timbres of different instruments to bring particular timbral qualities to their compositions.

With the invention of synthesizer technology, almost limitless timbral options have emerged, resulting in new ways of thinking about music. Timbre is a key element for Brian Eno which he describes as the "vertical colour of sound." Vertical music is heard vertically or spatially (Tamm 1989). The timbre, "colour" or mood of a piece are central features as opposed to tempo, melody and harmony. Ambient music can be described as a form of vertical music by bringing a timbral texture to the foreground and using it as the central material for composition. By contrast, Classical and popular music develops horizontally over time - in linear and often teleological (goal oriented) forms where listeners hear the music as a conversation, an argument or a series of statements. Vertical listening opposes this approach by inviting the listener to examine the timbral and spatial qualities of music in a way similar to examining a sculpture. Vertical music relies on subtle gradations and changes of timbre, tends not to have a regular pulse or beat, and often balances tone and noise. The listener can explore sound and space, over a long period of time, where attention is not directed by sheer volume or gripped by a virtuoso performance. The listener is allowed to absorb the music and can often embrace it as part of a larger lived experience, where every-day sounds can blend with the 
music. There is a choice to listen or not to listen, to focus or to ignore (Tamm 1989). This important aspect of ambient music was encapsulated by Eno (1975).

Ambient music must be able to accommodate many different levels of listening attention without enforcing one in particular; it must be as ignorable as it is interesting.

Eno's statement about the dichotomy between ignorable and interesting has almost become a slogan for defining ambient music. This fascinating idea takes various forms. For example, the shift from "interesting" to "ignorable" which is observed when an ignorable repetitive element becomes redundant to the listener but may become interesting again when varied or reintroduced. By constantly and subtly varying musical texture the listener's attention travels back and forth between ignoring the music and consciously listening. Eno calls this quality "ignoresting" (1978).

Western Art music composers in the 1970s were also exploring the realm of timbre. Spectralism is noteworthy in this respect. French composers Gerard Grisey and Tristan Murail are the most well-known of the Spectral composers. They were disenfranchised with contemporary concert music and felt composers had lost touch with audiences through the overly intricate works of serialism (Carey 2020). They were not inspired by the past, or by neo-Classical or neo-Romantic approaches to composition, and also rejected American Minimalism. They completely rejected tonality engaging with sound without major and minor triads, or tonal harmonic functions. Their view was that harmony, melody, counterpoint, orchestration, etc were outdated (Murail 1984). Spectralist composers declare that Spectralism is more than a style, it is a new way of listening to and understanding music much as is the case with deep listening. It is an aesthetic, a philosophy, and a way of thinking about musical composition where harmony and timbre have become two different facets of the same phenomenon (Rose 1996).

The origins of Spectral music are closely linked with the development of new computer technologies and in particular an electronic music technique known as additive synthesis. Additive synthesis involves the summation of component frequencies in order to build up complex composites (Rose 1997). Grisey and Murail became interested in timbre after viewing notes on a sonogram which allowed them to look deep into their acoustic properties (Bolanos 2020) (Rose 1984). Spectralist composers blur the distinction between harmony and timbre by composing with harmonics drawn from the harmonic series. This series established a vital point of reference for Spectralist composers. An early example of a work using the overtone series is Gerard Grisey's work Partiels (1975). The piece opens with the trombone playing a single low $\mathrm{E}$. The partials from the spectrum of this note were then arranged for the rest of the ensemble to play (Rose 1997). In this 
way Grisey was creating a hyper-intensified version of the original sound object the source note on the trombone (Bolanos 2020).

Some other techniques used in Spectralism are the use of extended techniques (including filtering techniques), subharmonicity and microtones (which derive from the upper reaches of the harmonic series). Extended techniques are used to create distinct spectral changes in the sound of an instrument. An example can be found in "Sept Papillons" for solo cello (2000) by Kaija Saariaho. She asks the cellist to play sol pontacello, bow up against the bridge, which amplifies the overtones making a spectral harmony and blurring the distinction between timbre and harmony (Rose 1996). Microtones are intervals smaller than a semi-tone. A piece known for its use of micropolyphony, micro intervals and extensive use of the overtone series is Georg Fredrich Haas's String Quartet No2 from 1998.

Reflection \#2 - Timbral considerations are part of my compositional process when making my ambient music. I create floating ambient atmospheres by using loops of sounds that have a warm, rounded, mid-frequency tone colour and have similar resonant properties as certain orchestral instruments. The qualities I am drawn to are the resonances of woodwinds, horns, vibraphones and bells. These work well together to produce the light, hovering character and suspended moods I am wanting to create in my impressionistic-ambient music. I have avoided obvious harmonic changes, form structures, and musical narratives or statements. The overall piece is the statement in itself. The intention is to draw the listener into the gentle tones of the music allowing the mind to move freely in connecting combinations of tones and timbres. Using a minimal sound palette and leaving spaces in certain registers, instead of filling the entire sound spectrum, I draw attention to the empty spaces between the notes, aim for less intensity, and focus attention on a gentle tonality which supports the mood or atmosphere I'm seeking to establish. Interesting sounds, pulses and loops can enter and exit the soundscape in a variety of ways giving the listener the impression that repeating motifs are like satellites drifting around a steady state background. (The steady state is discussed in the next section.) By using associated harmonic registers played by different sounds small ideas can gradually fade in or out, enter and exit through amplification of frequencies through EQ adjustments or automations, travel across the field or sit in a particular area using panning techniques, or sit in the background subliminally (barely audible) by using reverbs and panning. Working with a selection of synthesizer sounds can be challenging as the complexity of each sound can crowd out other more subtle sounds, but by altering the sounds in small ways I can give each a space to be heard. Changing the tone of a continuous texture or moving it back in the mix for a 
while, prevents it from becoming too tedious or dominant. It can be transformed to blend with newly introduced timbres or later reintroduced in its original form. A specific example from my portfolio with timbral matching and changing textures is Influx.

\section{Deep Listening}

Alongside the development of ambient music, the art of listening has changed into "astute listening" or "deep listening." Listeners have been encouraged to enjoy and explore the environmental sounds in ambient music. Pauline Oliveros was an ambient composer who believed the most considerate way to live is to listen deeply. In 1988, she descended 14 feet beneath the earth, into an underground cistern located in Washington, where sounds reverberated up to 45 seconds in the dampness. She took trombonist Stuart Dempster and sound artist/vocalist Panaiotis, to record music with her. The trio carried with them an array of items including an accordion, trombone, didgeridoo, garden hose, conch shell and a pipe, the sounds of which all became transformed as the bigness of the empty room morphed the sounds into something new. The music she recorded has been described as not of this world (Service 2012). Oliveros describes the music as an example of deep listening. It begat a new philosophy of the same name which focused on the possibilities of truly paying attention, retuning and calibrating your ears to allow for meditation and the preservation of well-being. Deep listening introduced into ambient music the radical possibilities of the body to overcome itself, just by listening hard enough (Alarcon 2017). Another interesting artist is Laurie Spiegel, a synthesizer composer who crosses boundaries between ambient and other electronic musical forms. Speigel also advocates for deeper listening through sonic awareness. The liner notes to her album The Expanding Universe (2001) asks " the listener to go deeper and deeper inside a single sustained texture or tone... The aesthetic aim is to provide sufficiently supportive continuity that the ear can relax its filters... The violence of sonic disruption, disjunction, discontinuity and sudden change desensitizes the listener and pushes us away so we are no longer open to the subtlest sounds. But with continuity and gentleness, the ear becomes increasingly re-sensitized to more and more subtle auditory phenomena within the sound that immerses us..." (Adkins 2019).

\section{The Steady State}

A vast array of music can be described as ambient (or ambient influenced) from Brian Eno's 1970s Music for Airports, consisting of tape loops, and a spacious but minimalist sound palette, to newer artists such as Jonsi \& Alex's whose Rice Boy Sleeps (2009) album features acoustic instruments alongside a string quartet, choir, electronic sounds, and heavily effected vocals. Despite this diversity one feature 
that appears to be common to all ambient music is a central component known as the "steady state." (Cummings 2019.) This is an element of the music that stays constant throughout and defines the fundamental behaviour of each piece. A composer can introduce small changes to other lesser elements within a piece while the static (unchanging) atmosphere continues uninterrupted in its consistency. The steady state may remain static or transform slowly over time. The overall effect of the steady state can be meditative and timeless, adding to the impression that the piece could go on indefinitely. This impression of infinity is reinforced by ambiguous phrasing as well as beginnings or endings that are "soft" rather than clearly defined. Ambient composers have created steady state textures using a variety of different elements including drones, repeated chordal washes, reverb effects, sound-stretching using granular synthesis, industrial and natural environmental sounds, ostinatos, a bass pulse, a time event, as well as non-musical noises and textures.

An early and celebrated example of ambient music, which serves as a prototype for the steady state compositional method is Brian Eno's Discreet Music (1975). The piece is an example of tape-delay montage where two short melodies interact on occasion on top of the steady state loops. Due to the use of different lengths of the tape loops, new textural relationships are constantly created as the loops never intersect in exactly the same way. Overlapping non-synchronised loops define the works fundamental qualities. The interaction of the loops playing through at different time frames produces music that is constantly changing which also suggesting that it could continue infinitely.

Taking the idea of the steady state further, ambient music could be defined by applying Fred Hoyle's steady state model of the beginning of the universe. Hoyle's steady state model claims the universe had no fixed beginning. It was always there and slowly grew and expanded. This of course is the opposite of the big bang theory. Ambient music shares attributes of Hoyle's steady state theory; as the overall behaviour of a core texture that is present throughout a piece remains in stasis, small changes take place in other elements. The steady state core anchors a piece in space and time allowing smaller elements to move, change and develop around it (Cummings 2019). Interplay between the steady state and other elements is also a characteristic of Eno's Discreet Music (1975). However, other composers had presaged the use of steady states well before Eno. An obvious example is Eric Satie's Vexations (1893) in which a repeating piano passage is played 840 times. Vexations relies solely on the steady state - in this case an ostinato texture - as a means to compose music intended to sit in the background, hence only able to be categorised as ignorable instead of offering interesting and new ideas to occasionally catch the listeners attention (Cummings 2019). 
Reflection \#3 - Examples of steady state can be found in much of the material in my portfolio, mostly through the use of ostinatos. I spend time making a modal riff in an impressionist style or from a particular chord I like, which I will often play in a modal way to avoid an obvious tonic. This acts as the steady state texture that continues throughout the piece. As I build the rest of the material around this steady state texture notes and timbres start to interact with each other through resonating in unexpected ways or cancelling each other out making a dip in the music. On occasion this "additive" process creates too many layers which later need to be thinned out. A particular example is The Range. A sequence of notes played on synth, based on a simple A major 7 chord, starts the piece. A bass ostinato joins the first texture, then other electronic elements and string-like materials drift in and out, sometimes playing in time and sometimes out of time with each other. The drift was created primarily in the string sounds by cutting out sections of a string motif and making a kind of loop, (although they are not strictly a loop as they are not all the same) then I rhythmically stretched them and placed them at irregular intervals with different fade-in times. Some of these string sounds appear to slowly emerge while others jump in fairly quickly. Another example of the steady state texture is I am Running. The background texture was created by delay feedback on the vocal line which produced a whispering effect. The effect was heightened by the use of the vocal harmonizer which gave it a haunting sound of multiple voices whispering.

\section{Vertical Time}

When we listen to ambient music initially our goal-orientated listening searches for a way to follow or understand a vertical piece which is not goal oriented (teleological) in nature. As we surrender this expectation, we can enter the vertical time of the work where music seems to evoke time (Kramer 1988). A well-known example of vertical time in the minimalist genre is Steve Reich's tape collage Come Out (1966). Two identical spoken lines drift out of sync until one is a full beat ahead of the other creating a rhythmic phasing effect. During the 20-minute piece, the listener has no point of reference and no way of telling if the composition will end or continue to infinity.

In ambient music vertical time and the steady state are connected. There are no dominant phrases leading the composition forward in time (teleology) or breaking the work into formal segments. In linear time by contrast, phrases group into cycles, subsections, movements etc in a hierarchical ranking with stronger cadences closing off sections of the piece. A vertically conceived piece has no set beginning or ending in that there are no events as such (Kramer 1988). Tension and release, 
to the extent that they exist in ambient music, are created through textural transformation. Pieces simply fade out or discontinue, avoiding dramatic endings. With an absence of harmonic progression, goal direction, formal hierarchy, and strong linear movement, an ambient piece is whole, undisturbed. The substance of the moment becomes the entire piece (Kramer 1988). Ambient works such as Terry Riley's A Rainbow in Curved Air (1969) show that phrase structure linearly connected events are not essential musical components. While phrase-like motifs emerge from the texture in Riley's piece, each cadence is equivalent in weight to avoid focussing or sectioning off parts. In this way the piece exists in its own (static) universe.

Reflection \#4 - An example of vertical time in my own work is The Vagus Nerve. This piece was recorded as I played it live in my studio and I've kept it as is to show an aspect of my composition process. This piece involves a series of ostinatos using the notes of a simple D minor 9 chord. The same notes are playing on 4 different synthesizers at different tempos. All of the sounds were chosen because they sounded like orchestral woodwinds and were within similar timbres. One sequence starts then others join gradually. (I see each sequence as a texture.) The changes taking place are through blending different combinations of textures or changing the textures themselves through EQs, ADSR, oscillation times etc. There are 24 possible combinations - I have only used some of the combinations. The Vagus Nerve has no linear events which would take the piece in a different direction. The synthesizers play on indefinitely while I experiment with changing small elements within the ostinatos. Towards the end I try adding vocals. These are in the background and although they sound accidental and different, they don't interfere with the non-linearity of the piece as the harmonics and phrase lengths are within the same boundaries as the other sounds.

Another example of vertical time in my music is Nothing I Want. This piece uses a pulse, and sounds similar to orchestral strings, flutes, and harps with the addition of vocals and spoken word. The pulse underscores the piece as the instruments change between an Ab major chord and a $\mathrm{C}$ minor chord. An ostinato loop starts and gradually disappears leading the listener into the piece. Short deconstructed string motifs swell in and out. The notes and harmony don't change. Timbral changes are heard throughout as fragments of motifs play in empty space by themselves or are combined with other textures. The vocal phrases cover a wide spectrum of registers. I've kept them minimal to avoid filling the entire frequency range and making too much density. They repeat as partial phrases or whole phrases. A small timbral change is created by a flute ostinato which increases in tempo over 
the piece and gradually becomes a pulse linking it with the starting pulse. At this stage the piece could start again and continue playing over and over. I decided to end it by having a spoken section. Overall, this piece feels like there are changes taking place, but there are no significant events or changes in linearity. The interest is created by small changes in the tempo, timbre or frequency.

\section{Chance, Generative Music and Infinity}

\section{Chance}

Many ambient composers incorporate a level of chance into their compositions, allowing music to emerge from chance processes instead of composing every note. As already discussed, in the 1970s Brian Eno was experimenting with tape machines in creating his ambient series. He describes this approach as unintentional and accidental. By allowing different lengths of tape to play simultaneously a composition was created by chance. Clusters of notes, silence, and sequences of notes forming melodies, appeared at random places (Tamm 1989). However, the composer is still involved in a variety of ways using careful listening to make small adjustments and decisions about the emerging music, as well as by making, selecting and combining the materials to be used in a piece. By setting up musical systems on reel-to-reel tape machines and analogue synthesizers and letting them run through, Eno and his collaborators were able to improvise over these textures. This allowed others to be involved in a collaborative composition process by listening and responding to the tape music. Side 1 of Eno's Discreet Music (1975) is an example of chance composition. While preparing background sounds on a synthesizer for a live performance, he was distracted by a series of events, which allowed the sounds to play through and record onto tape without interference. An additional accident was playing the tape back at a slower speed. He liked how the composition sounded and decided to keep it as it was.

Chance methods are not new, however. Composers have been experimenting with chance and systems-based composition as early as the $1700 \mathrm{~s}$. In the $18^{\text {th }}$ century the Musikalisches Wurfelspiel ("musical dice game") was popular throughout Western Europe. Several different games were devised using random numbers and dice. C.P.E. Bach is known to have used dice when composing Einfall, einen doppelten Contrapunkt in der Octave von sechs Takten zu machen, ohne die Regeln davon zu wissen ("A method for making six bars of double counterpoint at the octave without knowing the rules.") Mozart used a dice game to compose a number of waltzes in 1792. The method involved rolling dice to piece together a musical composition using an assortment of previously written small musical fragments. The earliest example of Musikalisches Wurfelspie/ was Johann Philipp Kirnberger's Der 
allezeit fertige Menuetten-und Polonaisecomponist (The Ever-Ready Minuet and Polonaise Composer 1757). Other later examples of chance methods are Stockhausen's aleatoric music and John Cage's indeterminacy. Karlheinz Stockhausen's aleatoric music is a form of improvisation which involves the performer improvising materials provided by the composer. The players choose to play in random order, a series of movements, chords, notes, or phrases that have been created by the composer. An example of this is Stockhausen's Klavierstucke $X I$ (1955). This solo piano piece consists of 19 musical fragments spread over a single page. The pianist chooses any fragment to begin the piece then proceeds through a labyrinth of other fragments. Once the first fragment has been played for a third time the performance ends. Cage used the idea of randomness or indeterminacy in many of his compositions. An example is Reunion (1968) which features chess players playing a game on a photoreceptor equipped chessboard. Every move triggered a different sound and distributed it to one of 8 speakers facing the audience. This means that each time a game was played a different piece of music was created (Alpern 1995).

Reflection \#5 - Chance plays a part in my own compositions. I create basic materials either by using a chord I like, for example the $7^{\text {th }}$ chord, choosing a group of the notes I like from Debussy, Satie or Tierson piano sheet music, or by accidentally finding notes that sound appealing together. The starting materials are related harmonically. I prefer to stay away from discordant notes as I am wanting the pieces to be able to be played in a relaxing home environment and be positive and uplifting. Allowing 2-3 sequencers to play together creates music I had not planned or imagined. Notes sound separately, or clusters of notes create interesting resonances together, or there are unexpected silences at seemingly random places. However, I am not working with different length tape loops and so it can be difficult to reach a level of randomness I am aiming for, and sometimes I realign sounds in the DAW to increase the sound of randomness. I am often pleasantly surprised by how it sounds when 2 sequencers play at the same time with different sounds and different tempos. This kind of combination often manufactures a composition. My role is to decide what sounds good and what doesn't, what can be kept, changed, and arranged. Or what can be improved by using production techniques such as changing filter settings, EQs and adding effects. Eno compares these techniques to being like a gardener, where one plants seeds and watches what grows. Unintended patterns are created by simply changing octaves, tempos, altering attack, decay, sustain and release. The challenge is to keep it minimal. I am trying to create music using a limited palette of notes and want to have silence in some registers. This is partly as a reaction against previous band projects where I was bored by all registers being filled with sound. When I decided to make music using 
modular synths it was a new medium for me. I wasn't sure how to use the technology at first and spent a lot of time trying to work out how to make sounds like the artists I admired. I tried following Youtube tutorials, which were often not successful. Eventually I came to a place where I would play creatively on the synths for my own satisfaction. The music did not have to sound like somebody else or have any commercial value. I decided on the sounds I like. I wanted to make the pieces "breathe," feel weightless and ethereal. Silence became part of the composition process. I described earlier, I usually play all of the patterns in the room and do live mixes experimenting with different ways to change each pattern before recording anything into Pro Tools. As I mentioned earlier The Vagus Nerve is an example of 4 different synths playing at different tempos without any interference from me. I liked how it sounded and decided to keep it in its original form, as a live demo, without making any alterations other than ADSR changes and the addition of reverb. This is a good example of how I might begin to work with chance music, although listening to it months after having created it I would like to thin it out. Another example from my portfolio is Murmuration. This is one sequence of random notes, rests and tied notes I programmed into the sequencer on the Moog. While the sequence played I made small changes to the octave rates and cutoff filter adding higher resonances which changed the timbre. I like the rich sound of the single ostinato in this one minute piece and how changing one filter can add some interest.

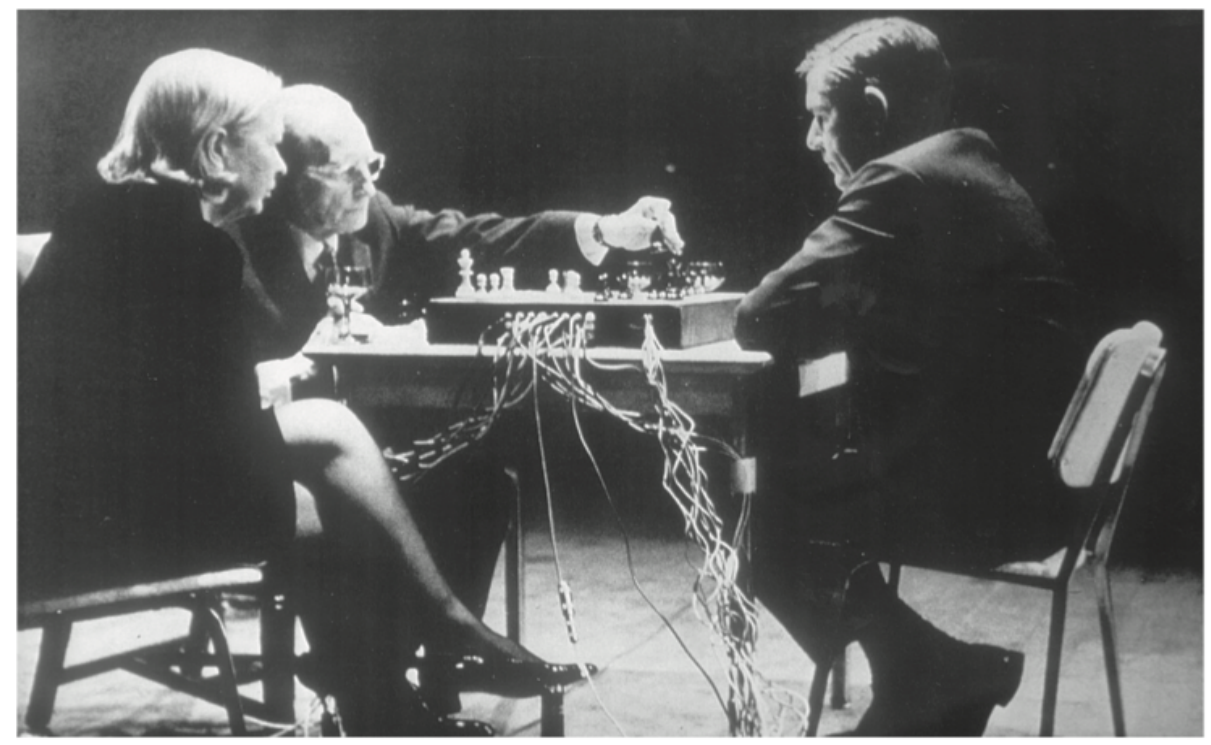

Fig. 11. Marcel Duchamp takes one of John Cage's pieces. (Photo: Shigeko Kubota)

Fig.2 Marcel Duchamp takes one of John Cage's pieces. (Photo: Shigeko Kubota) 


\section{Generative Music}

Generative music is the term used to describe a system when a composer uses an algorithm to put together a piece of music. Algorithmic composition was a term formerly used to describe music created solely by a computer using a series of procedures that lead to the generation of music (Simoni 2013) which has now be replaced by generative music. The earliest example of computer-generated composition was in 1955-56 at the University of Illinois. Lejaren Hiller and Leonard Isaacson programmed basic musical "raw" materials and stylistic parameters into an ILLIAC (Illinois Automatic Computer) computer which was then a high-speed machine. The programmers pre-arranged parameters were the basis for the computer to create a piece of music called the Illiac Suite (1955). The score was later transposed into traditional notation for a string quartet to perform (Maurer 1999).

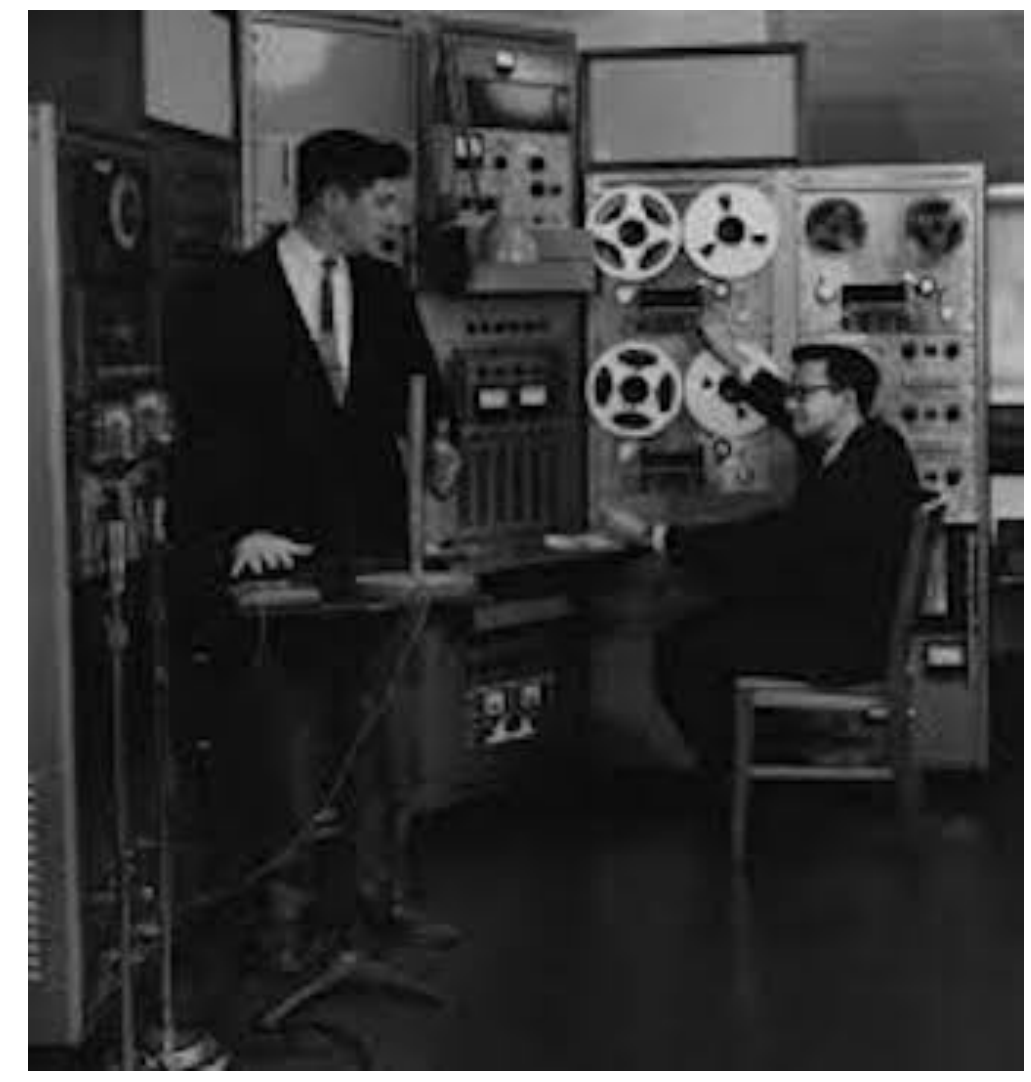

Figure 3. Hiller and Isaacson with the Illiac I at Experimental Music Studio, University of Illinois, 1955

Generative music is a system or algorithm employed to create part or all of a composition. Brian Eno coined the term Generative Music in 1995 when working on software for the SSEYO's Koan generative music engine. SSEYO became known through Eno's use of the Koan Pro software on his Generative Music 1 album (1996) which was released only on floppy disk. A specific soundcard was required to hear 
the music Eno intended. Without the soundcard the music of course sounded completely different.

The term Generative music has become widely used to describe any computergenerated music from completely random music mixes to rule based computer composition. Generative music is created by a music technology system where musical variables are set in place and allowed to run through to create a musical outcome. Eno's approach was influenced by cybernetician Stafford Beer's work on cybernetics and systems theory in the 1960s. It was first developed to focus on the homeostasis (steady state theory) of feedback loops that could regulate human body temperature. Beer uses the term "heuristic" to describe a set of instructions to search for an unknown goal, a process for scientific investigation, which Eno applied to his composition process. Beer's (1975) description is a useful encapsulation of the generative process whether used in music or other domains.

Instead of trying to specify it in full detail, you specify it only somewhat. You then ride on the dynamics of the system in the direction you want to go.

Eno claims that the two most important features of generative music are: 1) The music must continuously change and never repeat itself exactly: 2) It must be endless (Eno 1996).

Eno's principles of generative music are typically interwoven with other concepts described above, in particular chance composition, where a system is creating constantly changing music and the steady state methodology which allows music to continue indefinitely while other musical elements can change around it. The philosophy of sometimes focussing and sometimes ignoring the music is a logical reaction to music which never ends. Eno (2011) describes his generative compositional process as being similar to a gardener instead of an architect. An architect has the full picture of how something will turn out whereas a gardener plants a few well-chosen seeds and watches them grow into something of greater complexity. Eno (in Sherburne, 2017) explains how "gardens":

I have speakers everywhere, so I can have three-dimensional sound. I just start something simple [in the studio] - like a couple of tones that overlay each other - and then I come back in here and do emails or write or whatever I have to do. So as I'm listening, I'll think, It would be nice if I had more harmonics in there. So I take a few minutes to go and fix that up, and I leave it playing. Sometimes that's all that happens, and I do my emails and then go home. But other times, it starts to sound like a piece of music. So then I start working on it. 
Eno's Reflection project is a good example of his ambient generative work. It exists in a number of forms. As an album (2017) it consists entirely of generative music captured as one continuous track lasting 54-minutes. Eno also collaborated with musician and software designer Peter Chilvers to create an iOS app which plays the variations of Reflections. The app is coded to be a version of the album which is continuously recreating the music in different ways. It is designed to run indefinitely. Other forms of Reflections are less generative. A streamed version updates and changes every season. Hard copies are available on vinyl. The vinyl version is considered to be only one representation of the work (Sherburne 2017).

Reflection \#6 - My own work is more closely related to chance processes than generative music, although there are links to be found between the two. I use a method of setting up looped layers and allow them to run, resulting in something similar to Eno's "garden." However, my pieces do not play strictly as generative music as they are not constantly changing music that plays indefinitely. That would require the technology to be interacting in a selfgenerating way other than through the sequencer or arpeggiator. I am aware it is possible to repatch the signal flow to create random pitch sequences on the Moog, and as I have mentioned in earlier reflections, I have attempted to create generative music by learning the synth's interface and following online tutorials. However, I wasn't happy with the results and hadn't been able to make the kind of music I intended. Therefore, I started to compose by pursuing an alternative interest in repeating patterns, layering and field recordings. My main interest is finding new ways to be creative with music. Working further with generative music technology is still one of the sonic areas I am interested in exploring in the future.

\section{Atmospheres}

While ambient music has changed in style and scope over the past five decades the concept of "ambience" has remained essential to the genre. The terms "ambience" and "atmosphere" have become interchangeable in music and also in a variety of other fields such as architecture, colours and design, theatre scenography, art and also social settings.

In ambient music an atmosphere tints the listening environment creating a kind of perceptual haze that is difficult to define. Eno himself defines ambience "as atmosphere, or a surround influence: a tint" (Tamm 1995). Aesthetics theorists define "atmosphere" as a mood permeating a place, a situation, or a creative work. Atmosphere indicates something vague and indeterminate, but as part of everyday 
speech the meaning is clearer. For example, a garden can be described as having a tranquil atmosphere and black clouds as having an ominous atmosphere.

Ambience is connected ontologically to other compositional elements within the piece because an atmosphere is a totality, a holistic impression. We first perceive an atmosphere before discerning objects, forms or colours. For instance, when entering a room for the first time, one is immediately aware of a friendly atmosphere or a tense atmosphere (Boehme 1993). We are always in atmospheres and recognise the experience by emotional participation and awareness.

Phenomenologist Jurgan Hasse describes this as a 'felt sensitivity' through which atmosphere is perceived as 'instant totality' (Grant 2013). In Hasse's (2011) words "places with vital qualities that can be felt sensitively, cannot be analysed gnostically, but are to be perceived in their instant totality as atmospheres...we relate ourselves to them, or live in them..."

Phenomenologist Hermann Schmitz provided a philosophy of the body to explain the concept of atmospheres by linking space with emotion, instinct and impulse. The social aspect of togetherness is a way to explain a shared corporeal experience as we perceive atmospheres. Atmospheres are without boundaries and our bodies interpret them as real. The body receives an atmosphere as a mood, emotion or a visceral feeling. Other objects, colours and even people are perceived after the atmosphere (Grant 2015) (Boehme 1993). Atmospheres can reflect the sounds of a particular place or conjure up a memory of another time. Atmospheres which reflect a melancholic state of mind can be reproduced to communicate the same mood to the listener (Bohme 1993).

In ambient music atmospheres are presented like impressionist environments utilising a restrained sound spectrum that makes use of the steady state, alongside longform composition, and electronic sounds. Ambient atmospheres can be created by layering sounds, creating textural soundscapes, collaging and sequencing any variety of elements, or using the sonic ambience of an abandoned building, an industrial or natural soundscape. Atmosphere can be about filling space or opening up a sense of space. Brian Eno's seminal work Ambient 1: Music for Airports (1978) is an example of atmospheres that open up or create space. This work uses a repetitive tonal harmony, an absence of abrupt attacks, long decays, a minimal number of textures (1-2) and reedy sounds present at one time, and nonteleological writing. This leisurely paced, undemanding music has no clear foreground and listeners are free to explore the music, their own thoughts or let sounds merge into the background environmental (Demers 2010).

Before Eno, Pierre Schaffer and Karlheinz Stockhausen were both early pioneers of using collaging and other techniques to evoke various atmospheres. Stockhausen's 
Gesang derJunglinge (Song of the Youths) (1955-56) is recognised for early use of space and atmosphere (Prendergast 2003).

A specific character of Brian Eno's music is the use of a single atmosphere which pervades the music. The atmospheres are unobtrusive and emotionally neutral inducing calm and a space to think. An interesting example of atmosphere is again Side 1 of his Discreet Music (1975). Consisting of a small number of randomly occurring synth notes using warm, rounded sounds similar to heavily reverbed clarinets or flutes, the 30-minute piece feels like it could continue endlessly. The emotional character of the overall sound is meditative and emotionally ambiguous. Another interesting example is Eno's album Thursday Afternoon (1985) which is characterised by a subtly changing continuous background atmosphere interspersed with reverberated piano notes that float over and through this atmosphere (Adkins 2019).

Reflection \#7 - When I first started making ambient music, I felt free to create the moods and sounds that only I wanted to hear. It was refreshing to be away from songs, guitar distortion, drumkits and the compromises that come with being in a band. I felt it was time to move onto something else although I didn't know what. I began making open-ended pieces that relied on an atmosphere without structure - an escape from song writing. The atmospheres I created evoke a feeling of calm introspection and although they are somehow optimistic there is also a slight haunting feeling. I wanted to bring in my interest in retro and analogue instruments and try to invoke a feeling of nostalgia by using harmonies that suggest a mood reminiscent of orchestral scores from old movies. I use warm synth sounds, similar to the reedy sounds of clarinets or flutes and combine these with piano loops and occasional vocals. Minimalist piano sounds seem to evoke an identification with a solitary figure giving the music a lonely feeling, which adds to the atmosphere. I have used this piano in this way on I am Running. Other examples of atmospheres in my portfolio are The Range and Influx. The Range has a gentle, contemplate mood created by the floating strings which drift in and out. I intended the piece to have no foreground or teleological development. Nothing happens in terms of linearity, there are no events or statements. The harmony remains confined to a series of notes of an $A$ major chord. Two ostinatos play constantly in the background. I wanted the listener to ignore the ostinatos when the ethereal strings are playing and be aware of them when the strings stop. Heavily reverberated Vibes intermittently play a 3-note motif. These have a soft upbeat feeling which stops the piece from sounding too sad. The overall effect is calm, unintrusive and relaxing. A sound world where there are no events or statements. A comforting road to nowhere. Influx has a languid atmosphere 
like a lullaby where shimmering strings hover and float about merging with indecipherable phonetic poetry and a series of repeating notes and soft, delicate sounds. Two ostinatos start the piece playing at different tempos. The reedy fast ascending and descending ostinato is on the Moog, the other ostinato is the same notes played by a super slowed down vibe sound on the Korg micro. In the background you can hear resonant frequencies created by the reedy Moog sound which seem to create a sustained wall of mid-range resonating frequencies. This was created by a longer release filter and a large amount of reverb effect on the synth. When the vocals enter (sung through the vocal harmonizer) they take a central place and feel like they might sing a song, but it becomes obvious they are chanting something indecipherable. Gentle fuzzy strings emerge replacing the vocals and a glissando drum hit created on the Moog Mother 32 join in. By splicing up the vocals and placing them at the end in random places (visually using the Pro Tools grid to ensure they were not lined up evenly) made a nice ending without changing the harmony or adding new material. This piece is upbeat but also haunting.

This chapter has defined ambient music specifically through features that differentiate it from other genres. These essential features are timbre as a central element, music that can be in the background or in the foreground through deep listening, music that has a lack of drumbeats, harmonic progression and formal development and the presence of a steady state texture that is continuous possibly lasting to infinity. The presence of a particular atmosphere is another central feature of ambient music and the incorporation of any type of musical or non-musical sound. Other special features in ambient music that are closely related are chance composition and generative music processes.

The next section will firstly explore and discuss methods and processes used by ambient artists, by focussing on specific textures that are widely used in ambient compositions. The second part will look at what presaged ambient music by discussing composers and ideas that influenced the ambient genre.

\section{Making Ambient: Methods and Processes}

This section explores different methods and processes that ambient composers use to create music, and also looks at artists who have influenced the ambient genre through their work.

A key technique used by ambient composers is making background textural elements a central focus in the music. This is in contrast to other musical genres where melodies, harmonies and rhythms are centre stage (Bates 2019). Texture has 
also featured as a key component in New Age, alt-rock, electronic, metal and indierock music since the 1960s. This indicates the extent to which "texture" music had become a concern across a broad range of musical forms. However, a wider consideration of texture is beyond the scope of my exegesis.

In ambient music a central textural element may be presented as the piece. This texture transforming as a development or be layered with other sounds which change around it. Transformations which unfold over time is the most effective way for listeners to hear changes in the texture while exploring the resonances of spaces or the resonances of sounds (Demers 2010). Textures which are prevalent through much ambient music are drones, loops, and environmental noise. These link in with the steady state by continuing throughout a piece and can affect vertical time by slowly evolving. Our perception of time is changed by exploring these textural elements. In the next section I will examine these distinctly different textures and discuss how they have been used by composers. I will also forge links with my own work.

\section{Drones}

Drones are textures which use sustained notes or sounds that continue uninterrupted for long periods of time. In electronic music drones can be created in different ways including through synthesizers, guitar effects pedals, DAW software, tape loops, and field recordings, such as the industrial drones or nature sounds. Drones are not the exclusive domain of ambient music. The sustained sounds of drones are sometimes built into acoustic instruments such as bagpipes and the hurdy-gurdy. Indian Classical music and Tibetan ritual music are just two examples of the use of drones in ancient Eastern cultures. In Western culture audiences first experienced powerful drones through Medieval Catholic chants sung in reverberating cathedrals, and through the drones of early organ music (Boon 2019). An example of early organ music performed on a portative organ (a small upright keyboard instrument) is The Robertsbridge Codex (1360).

From the late 1950s onward drones were in the work of American minimalist composers such as La Mont Young, Terry Riley and Pauline Oliveros. Drone-based music can be understood as a reaction against mid- $20^{\text {th }}$ century musical modernism and traditional forms of composition which remained concerned with more traditional musical elements and processes. A representative example of a composition using drones is Young's Arabic Numeral (1960) for piano(s) or gongs(s) or an ensemble of at least 45 instruments of the same timbre. The performer/s use a directed improvisation method, which starts with a single drone or drone cluster, and the performers decide how long each drone will be played for and how many repetitions of the drone to play (Tote 2010). 
When creating drone-based music Young, Riley and Oliveros used any combination of influences they felt fitted their work, including aspects of Indian classical music, Zen Buddhism, yoga, Tai Chi Chuan, and Arabic music. The works could be for a single drone, a complex of drones or a sustained sonic texture. Their focus was on the "here and now" of sonic experience. This idea reflected an interest in Eastern spirituality and alternative religions which was emerging in American culture in the 1960s. Further significant examples of drone music are La Mont Young's the Compositions (1960), Terry Riley's In C (1964) and Pauline Oliveros Sonic Meditations (1971-7). An extreme example of drone music is La Monte Youngs extended piece for sine-tone waves and lighting effects The Dream House, (1960). Installed in a New York loft, as a continuous environment intended to run for eight years or possibly forever (Prendergast 2003).

More contemporary forms of ambient music also influenced by newly available technologies are Dronecore, Dark Ambient and "isolationist" music. These ambient genres also employ drones extensively. Dark Ambient uses discordant timbres to produce a dark, ominous mood. The music often includes low rumbles, industrial machine noises and dissonant harmonies. An example of dark ambient from 1978 is Throbbing Gristle D.o.A: The Third and Final Report of Throbbing Gristle. A more recent example is Glaswegian Kevin Doherty's project Sleep Research Facility. SRF make spacious soundscapes of richly textured layers of manipulated noise and mechanical drones. The beatless music is intended to function as a sleep aid as well exist as a musical work. The music avoids sudden changes which would disturb sleep allowing the listeners thoughts to gently drift. The liner notes state, "Recommended playback level: just above quiet." This statement links directly with Eno's ideas about ambient music being dynamically low, not imposing itself, mixing with environmental sounds, and having blurred edges and washes of colour (Tamm 1989).

Drones have also been used in a myriad of rock music genres over the last five decades. Drones were first heard in rock music in 1966 when contemporary rock musician John Cale played a viola drone on the Velvet Underground song "Heroin" (Schwarz 1996). German Krautrock bands in the late 60s and early 70 s were experimenting with repeating patterns and drones as a reaction against American Blues music, and old-fashioned German pop Schlager songs. Krautrock bands Can and Neu! created long-form improvisations with the use of continual unchanging motoric beats that acted as a drone, while other repetitive patterns were layered over top with guitar, synthesizers, and voice, creating drone upon drone (Tote 2010). In the 1980s New Zealand's Flying Nun bands adopted a drone aesthetic creating an entire music scene centred around a drone style called the "Dunedin Sound." A key example is The Clean, arguably the most influential band on the 
Flying Nun label. On Tally Ho (1981) an electric organ drone underpins the entire song (Shepherd 2016).

Reflection \#8- My own interest in drone music stems particularly from my work with San Francisco based shoegaze band Orange in the 1990s who were part of the same music scene as Brian Jonestown Massacre. Orange based our sound on layers of textured guitar drones, heavily effected by chorus, reverb and delay pedals, combined with reverbed vocals. We leaned towards creating harmonic overtones covering a full spectrum. One tuning I favoured which supported the generation of overtones was to tune all my guitar strings to $E$ and $B$. I then ran the guitar through a chorus and/or delay pedal to double, blur and soften the notes, and added distortion to give a unique grittiness to the sound as well as emphasise higher harmonics. Interestingly, in my current research, I have become aware of La Monte Young's use of a prolonged drone interval of a fifth in his Composition 1960 \#7. He describes the two-note chord as "allowing all sorts of harmonics to hover in the air, adding a quivering resonance to the apparent stasis" (Schwarz 1996). This is a quality also evident in Orange's music, which listeners often described as shimmering and sparkling, within a warm wash of sound. I was also lead vocalist in Orange. I ran two microphones, one with delay, the other with reverb, as a way to blend the vocals into the guitar textures instead of a conventional mix where the vocals are loudest.

In my current work on modular synthesizers, I see drones in a refined way using ostinatos as a continuous textural drone or layering drones upon each other to create density as a timbral development. This gives the effect of a drone if played at

a low volume. The repeating patterns are modal implying a key centre staying within a static harmonicity. This music is intended for domestic use to accompany activities such as cooking, bathing, yoga, studying, daydreaming or reading a novel. As a way to counteract the gravitas of covid19 lockdown my intention was to avoid doomy darker sounding music and aim for something unintrusive yet subliminally uplifting.

\section{Loops}

Loops create a repetitive rhythmic patterning which can be layered to form dense textures of sound or used sparsely for minimalist compositions. Tape loops have been a central feature of electronic and ambient music since the 1970s. However, as earlier in the $9^{\text {th }}$ century there is evidence of mechanical music technologies playing cyclic music. One example is the Banu Musa, an automatic flute playing machine developed in Bagdad at the turn of the century, which played a series of repeating notes via a hydraulic system (Levaux 2017). While there were many such devices invented, it is beyond the scope of this essay to discuss this further, but 
this example does provide evidence of the longevity of "looping" practices. Instead, I will focus on composers who used repetition in their music, and in particular tape loops. Tape loops were used by many contemporary electronic composers from the 1940s onwards. Pierre Schaeffer is the first person in Western art music known for composing music using the concept of a loop. In 1948 he noticed how dust and scratches on a gramophone disc made the needle skip back to a previous position, creating what he called a "locked groove." Schaeffer began experimenting with engraving closed/locked grooves on shellac discs to make music which he called "musique concrete". The first tape loops were created in the early 1950s when Schaeffer recorded sound onto magnetic tape which he then spliced up and joined end to end. The tapes could be played continuously (Levaux 2017). In the 1960s, minimalist and experimental composers including Eliane Radique, Karlheinz Stockhausen, Steve Reich, and Terry Riley used loops to compose a wide variety of pieces based on repetitive patterns, textures and looping rhythms. Riley's first loop composition M Mix (1961) is an interesting example of early experimental methods using magnetic tape. The raw material consisted of piano notes, people talking, and found sounds, some of which were distorted. Riley's small studio was in his home garage. Some of the loops were 30 feet long and had to be wound around wine bottles out in the yard to hold them in place while they fed through the reel-to-reel tape machines. Another interesting experiment with tape loops is Steve Reich's Livelihood (1964), where he hid a microphone in a NY cab and recorded conversations unbeknownst to the passengers. He created loops from the recordings using speech fragments, automotive sounds and slamming doors, then combined them into a 3-minute piece. Another example is Terry Riley's Poppy Nogood and the Phantom Band (1967) which consisted of brief melodic modules that are repeated and layered (Schwarz 1996).

As l've shown tape loops were in use well before the release of Brian Eno's Music for Airports (1978). Eno was the first composer to bring loop-based music to a broader public. By bringing techniques from minimalism, as well as chance methods, into his work using the same techniques as Riley, Cage and Young, Eno employed looping and chance as a reaction against the macho rock ethos that was popular in the 1970s, much in the same way as Riley and Young reacted against the formalist complexity of Serialism in the 1960s.

A very important feature composing with loops is evident in the work of Brian Eno who understands the repetition created by loop techniques as being like automatic writing (writing produced involuntarily when the subject's attention is directed elsewhere) where an element repeats and becomes irrelevant allowing the true ideas to surface (Tamm 1989). Notably the term "automatic music" was used by minimalist Steve Reich a decade earlier to describe his composition processes. Eno's compositional workflow involves letting work-in-progress play while he is 
involved in other tasks. Playing material "on loop" as a background allows him to be a listener - an activity which feeds back into further compositional work. By keeping busy with everyday tasks, Eno maintains that he prevents himself from adding more into the music, and in this way, he stays true to the parameters of a minimalist ethos in which the essential elements of a composition, its "true ideas" are not obscured. Eno switches between approaching music as a background element and as a central focus, allowing his role to shift between that of a composer and listener Sherburne (2017). Eno (2017) sums up his listening-based approach as follows: "As a maker, you tend to do too much, because you're there with all the tools and you keep putting things in. As a listener, you're happy with quite a lot less."

Terry Riley (1961) articulates similar ideas when noticing the change in his own perception while listening to repeated sounds, a process through which he becomes aware of different sonic and musical qualities.

I think I was noticing things that didn't sound the same when you heard them more than once. And the more you heard them, the more different they did sound. Even though something was staying the same, it was changing. I became fascinated with that. I realised it was stasis - it was what La Monte and I had talked about a lot in terms of his long-tone pieces - but it was stasis in a different application. In those days the first psychedelic experiences were starting to happen in America, and that was changing our concept of how time passes, and what you actually hear in the music.

This recalls Pierre Schaffer's comments, made in the 1950s, on repeated sound: Repeat the same sound fragment twice: There is no longer event, but music" (Schaffer 2012).

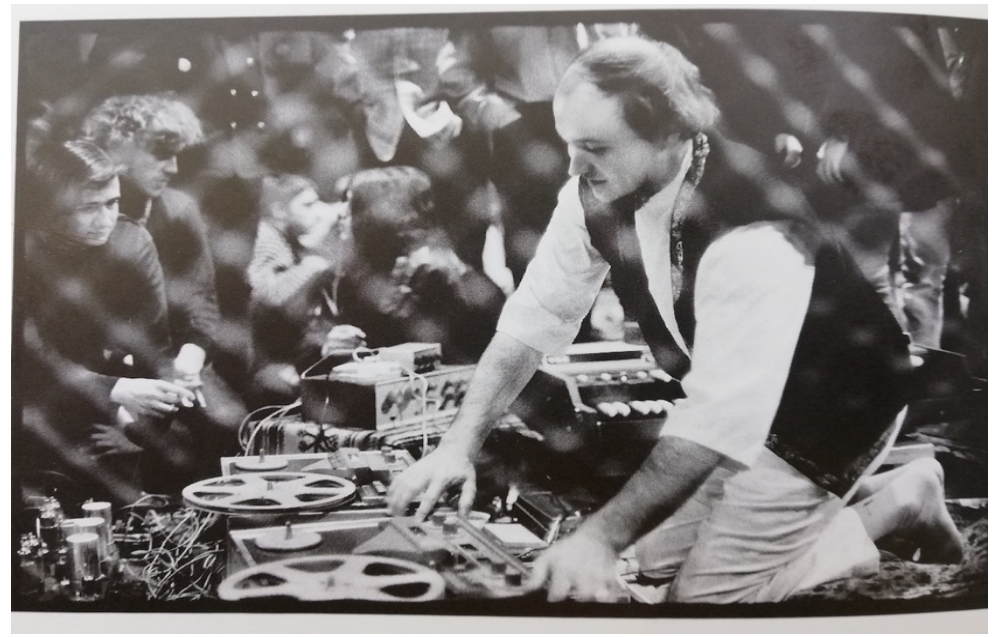

Figure 3. Terry Riley making live tape-music using 1968's version of state-of-the-art portable tape recorders. Photograph by Rob Benson, courtesy of Terry Riley. 
Conventional composition process involves developing ideas and refining materials before a piece is completed. By contrast, when working with tape loops a different kind of creative process, as well as a high degree of chance (when loops are layered) is in play, allowing the composer to become a listener, an arranger and a producer. These are different skills compared to those of conventional composition and may involve removing materials instead of developing them or simply becoming attuned to the music as it is. Loops can be allowed to play at random or set to run at specific times. Electronic loops are obviously more versatile than working with metres of magnetic tape and can be altered in the DAW with special effects or copied for an unlimited number of iterations. Eno's experiments with tape loops, especially the processes he used in Music for Airports, has been well documented and illustrates the link between using loops and the listening-informed approach to composition discussed here. Eno describes his process as follows:

The particular piece [Ambient 1 from Music For Airports] I'm referring to was done by using a whole series of very long tape loops, like fifty, sixty, seventy feet long. There were twenty-two loops. One loop had just one piano note on it. Another one would have two piano notes. Another one would have a group of girls singing one note, sustaining it for ten seconds. There are eight loops of girls' voices and about fourteen loops of piano. I just set all of these loops running and let them configure in whichever way they wanted to, and in fact the result is very, very nice. The interesting thing is that it doesn't sound at all mechanical or mathematical as you would imagine. It sounds like some guy is sitting there playing the piano with quite intense feeling. The spacing and dynamics of "his" playing sound very well organized. That was an example of hardly interfering at all.

Looping remains a vital technique in ambient music. A notable example is William Basinski's The Disintegration Loops (2002) which began thirty years before its final inception. In 1980 he started making tape loops using a variety of sounds including recordings of his own piano playing, birds chirping, coins dropping, others from accidental noises or from radio-station broadcasts bleeding into his amplifiers and tape decks. "I was getting all this great stuff... never could have created with pencil and paper. It was just coming from the sky" (Basinski 2014). In 2001 he began transferring his 1980s archive of loops onto digital format. Many of the magnetic tapes were in a state of decay flaking and falling apart as he let them play through the decks. The recording of this process became The Disintegration Loops. Basinski's innovation, on top of the familiar use of loops, was to capture the physical change and age of the tape medium. One of the defining factors in The Disintegration Loops is the fact that machinery is in a process of failing, and that this change itself is a key element of the composition (Frere-Jones 2014). 
Reflection \#9 - Some looping techniques I use in my own work involve creating loops on synthesizers (technically these are ostinatos), creating loops in Pro Tools, and using an loop pedal to create piano and vocal loops. When creating loops on a synthesizer I use the sequencer and the arpeggiator. I mainly use the Moog Grandmother (MG) for programming sequences as it has a 256 step sequencer that is very versatile and allows me to add rests, ties, accents, notes of different lengths, and play additional notes during playback, without having to call up a window on a screen - everything is tactile and immediate. One experiment I tried was programming the 256 steps with different notes and note values, rests etc all within the same harmony, then slowed it down. I had intended to make a long piece that never repeated. (Unfortunately the piece became too long and difficult to manage. I abandoned it before it was completed.) The arpeggiator simply plays a sequence of notes in order up to 3 octaves and is good for creating swooping cascades of notes and building a rhythmic base. I used both sequencer and arpeggiator on my pieces creating different layers to later experiment with in the DAW. The Moog Mother 32 (M32) synthesizer has a more limited sequencer at 32 steps. The two Moog synthesizers work well when playing sequences together, one at a different tempo to the other. My preference is to set the M32 to run at a slower pace against the GM sequence and then stand back and see where the notes "land". I can experiment with small adjustments to the tempo, ADSR or oscillators until I hear something I feel is interesting. On Coral Bleach the first loop is a fast 1 octave arpeggio making a pulse-like sound as a base texture, the second is the same pattern of notes slowed down and set on 3 octaves, making a cascade. I briefly bought in another synth, the Korg Micro which plays a loop using only 3 notes from the original pattern. The string ostinato was made on the Waldorf blofeld for a change in timbre. The blofeld contains 780 sampled sounds of synthesizers from different eras. Once I find a sound that matches or contrasts with the others in timbre, the patch can be altered to suit. Originally the string loop was a longer idea. I cut it in half in Pro Tools creating a loop then placed it sparingly towards the middle of the piece. Then reduced the strings loop further to make another patterns for the Coda. All patterns float in and out of the sound field.

I use an loop pedal for creating piano and vocal loops. One piano loop plays on track 10 Mixed Environments. I played the piano motif in two different octaves then released the loop to keep repeating it. The base loop is a sequence on the Moog GM. The woozy sound is the Moog M32 malfunctioning - I liked it but it had to be altered in pitch shift in Pro Tools. The vocals were sung using a voice harmonizer. The vocals were later cut into loops, treated and stretched in Pro Tools, then placed in the sound field. 


\section{Environmental Sound and Noise}

For the purpose of this exegesis, I will start from the $19^{\text {th }}$ and $20^{\text {th }}$ centuries when historians wrote about the use of environmental sounds in music composition. These sounds were reflecting the new noises of the industrial world and were initially referred to as noise. Noise is culturally constructed and is often interpreted as a disturbing element that compromises our comfort within the sound world. Yet what was once considered noise can become familiar and accepted as something that is not noise anymore. For example, the sounds of another language being spoken can be described as noisy.

Noise was first thought of as different from the sounds of the natural world and the sounds of music associated as it was with the industrial revolution in the 19th century and thus with the working class, disorder and the exterior world of the city. The upper classes by contrast preferred to be locked away safely inside, which includes listening to music inside the concert hall. Any external sounds could spoil the audience's experience of music and was for this reason considered unwanted in these environments. The only noise allowed was regulated sonic participation in the form of appreciative applause (Hergarty 2007).

Humans can be affected by certain noise frequencies; high frequency noise can damage hearing while low frequency noise can affect digestive and heart functions. (Prendergast 2003).

Noise was first introduced as a musical aesthetic by the Italian Futurist movement leader Tomaso Marinetti with his notion of "sound poetry." Described as a verse without words, "sound poetry" uses phonetic aspects of the human voice instead of semantic and syntactic features. In 1913 the idea of noise music was introduced with the publication of Luigi Russolo's "Art of Noises" manifesto. As a Futurist, Russolo urged people to embrace a new music based on the wider world of sound, claiming the only difference between music and environmental or artificial sounds like trams or crowd noises, was the composer's intentional arrangement of these materials. Russolo was interested in noise composition and ideas of randomness well before Pierre Schaffer started his work with musique concrete. In 1914 Russolo developed "noise machines" the intonarumori. These looked like wooden boxes with megaphone horns which made rustling, burbling and detonating sounds - at times referencing sounds commonly heard in WW1 (Prendergast 2000.) The machines were intended to replace the traditional orchestra with ensembles of these noise machines. Two pieces he composed and performed using the intonarumori were A Meeting of Motorcars and Aeroplanes (1913) and The Awakening of a City (1914.) Although the intonarumori were intended to replace traditional musical instruments The Art of Noises shows a consideration for the 
listener in recognising that noise music would be necessarily different to other music (Hegarty 2007).

Another early sonic explorer experimenting with the concept of environmental noise was Charles Ives, an American composer and philanthropist. He used incongruous environmental noises in Central Park In The Dark (1906.) During the haunting sevenminute orchestral piece a brass band suddenly breaks in on an eerie quiet section then disappears back into the music (Prendegast 2003). The next important moment in noise history after Russolo and Ives comes with John Cage's work 4'33. Cage advocated for all sounds to be considered compositional material. After a visit to an anechoic chamber where the only sounds he heard were nerve operations and blood circulating in his own body, he was inspired to compose the silent piece 4'33. In which the audience's attention is drawn to formerly unwanted environmental sounds. As a listening intention Cage was able to encourage the audience to observe environmental sounds while they sat in silent expectation that something else - conventional music - was going to happen (Hegarty 2007.) In 1975 Brian Eno merged environmental noises and music inventing the term ambient music, as I have already discussed. Eno's use of environmental sound has been highly influential in popular electronic music genres. For example, during the 1980s London rave scene pioneers The Orb, created Ambient House music using noise as a central element. A good example of this is their composition Adventures Beyond the Ultraworld (1991) (Prendergast 2003).

The inclusion of environmental sounds and noise is one of the main elements that differentiates ambient music from other music genres, and can include spoken word, found sounds, any type of environmental noise found in built or natural settings, synthesizer sounds, guitar feedback, sounds produced by DAW processors, editing tools, sound morphing plugins, and glitches caused by technological malfunction. Noise can be any element that is not conventionally musically focused on equal tempered pitch and metric rhythm, but it can also apply to "unmusical" sounds made by musical instruments such as squealing distorted guitars and non-musical sounds of the human voice. In ambient music noise can be used as slowly evolving textures or as sudden interruptions, as real-world or artificial sound environments. In non-ambient music noise is a negative reaction to sounds that are considered unwanted or annoying.

Japan has its own history of ambient composers. It would require an entire exegesis to cover the genre within Japan, but it is worth mentioning one distinguished composer. Hiroshi Yoshimura, born in 1940, and considered a pioneering musician and composer of ambient music in Japan. He was a leading artist in environmental music, layering together soft electronic melodies and the sounds of nature. Yoshimura made music for galleries, museums, building spaces and train stations. 
His most influential albums are Green (1986) and Music for Nine Postcards (1982) are considered two important albums in Japanese ambient music. Thanks to YouTube's algorithm, Yoshimura's music has been rediscovered and is now appreciated by thousands of new young fans (Yeung 2018).

"I will be happy if, when you enjoy this album, the surrounding scenery can be seen in a slightly different light." Yoshimura (1986)

Reflection \# 10 My piece Koi Pond contains field recordings of water sounds I collected in Fiordland. This piece was written as a reaction to the way humans use animals for entertainment, in particular applying to the Koi Pond at Changi airport in Singapore - a stark black tiled pool containing many Koi carp who swim in endless circles within an environment devoid of any natural features of the wild. I have inlaid a loop of water trickling in a small cave, this sound has a special kind of intimacy and tonal quality created by the rock walls of the cave and the shallow depth of the cave, (which incidentally was surrounded by moss, ferns and other greenery unlike the pond at Changi.) Later the piece is completely overwhelmed by a hissing whitenoise sound of fast running glacier-melt water - also recorded in Fiordland. The music is a somewhat cliched oriental in its choice of notes - the pentatonic scale the ostinato loop plays on endlessly representing the unfortunate lives of the trapped fish. This also links in with the ideas of infinity that Brian Eno talked about when describing the main features of ambient music.

\section{Concluding Thoughts}

Ambient music can be identified as music that is gentle and unimposing and able to accommodate many levels of listening attention without enforcing one in particular, with a focus on timbral exploration through longform composition. At its core the music tends to feature a steady state texture existing as an atmosphere that could continue indefinitely, with other coexistent elements. Ambient music is difficult to describe as it seems to float and drift as a gentle atmosphere tinting the environment around the listener which allows it to combine with environmental sounds. There are no significant sonic musical events or signposts in ambient music allowing the listener to explore the music or settle within their own thoughts. While these concepts might seem unusual in our age of multiple distractions the influence of Brian Eno's (1978) philosophy of ambient music, that it must be as ignorable as it is interesting, still has relevance for composers and listeners and indeed the popularity of ambient would suggest it has an important place in contemporary music. 
Interesting factors at play for the composer of ambient music are the use of generative techniques and a level of chance composition which contrasts with traditional methods of composing music. These unconventional methods require a different kind of composer to evolve; one who is a listener and a creator, an arranger and producer, and a collator or curator of sounds. It is also a collaboration with technology, through using algorithmic systems to compose part or all of the music. This is an act of chance composition where accidents are allowed to exist and be used as materials for creating music. The composer works with the materials produced through such processes.

\section{The Future of Ambient}

Ambient music has presented a way to compose and listen to music that is very different from other musical genres and yet ambient music is still developing as new artists bring different and original ideas to the style.

Since the inception of ambient music many associated forms and genres have developed. These include: environmental music, deep listening practices, ambient techno, ambient dub, electronica, electronic listening music, isolationist, postindustrial ambient, space music, brainwave music, picture music, ambient jungle, steady state, holy minimalism, Fourth World, New Age, chill out and new music (Toop, 1995) as well as the more recent genre of pop ambient. Technological developments and new musical trends continue to expand the genre, which at this point is very broad genre indeed.

Musicians following the initial emergence of ambient in the 1970s, continue to experiment with and redefine the ambient genre, using and exploring the techniques and ideas introduced in this exegesis: steady state, generative systems, atmospheres and so forth. A good example is Liz Allen's pop ambient work as Grouper; on AIA: Alien Observer (2011) she uses only voice and guitar to create ambient music which melds with popular practices. Ambient Techno artist Wolfgang Voight's project GAS is another example. On Pop (2000) track 4, he uses one unchanging slow drumbeat combined with natural water sounds to establish a steady state atmosphere. The slow beats challenge established ideas about ambient being beatless.

My own take on ambient music has been to use various ambient techniques such as gently floating textures, repeating loops or ostinatos, drones and chance approaches to composition. More personally, I have built on the ambient genre in my own practice by creating a group of works which encompass historic elements of my life in music. These elements include an interest in modular synth music, 
Impressionist harmony, minimalist ostinatos such as those used by composers like Philip Glass, woodwind and string motifs influenced by film music, and vocalisations reminiscent of indie-pop. While I have listed examples of music in this exegesis that have interested me, I have also been influenced by modular synth artists Kaitlyn Aurelia Smith, Caterina Barbieri and Suzanne Ciani, 1970s experimental German rock and electronic music ( Klaus Schulze, Popul Vuh, Kraftwerk and others ), Harold Budd, and Yann Tierson's piano music, Steve Reich's expansion/protraction processes of doubling or halving a phrases, Paul Lansky's vocal layerings, and Lauri Anderson's spoken vocal pieces, to name but a few.

The works I have composed for Inside the Microcosm, my MMA project, are intended to be used within the home to accompany domestic activities such as bathing, cooking, sleeping and daydreaming. My intention is to create gentle unassuming music which can be in the background or foreground, ignoresting music in other words. However, the pleasantly unobtrusive sounds allow one to reflect on external concerns, particularly global warming in the wider world.

Examples of these concerns are in the track titles; Coral Bleach, Influx (of meltwater caused by glacier melting,) The Range (refers to a chart showing ambient air quality.) Processes known to combat environmental destruction are also addressed: Rewilding (restoring to an uncultivated state) and Chlorophyta (a type of green algae adapted to living in extreme conditions) are a few of the ideas that interest me outside the world of music.

These pieces are steppingstones to future work, including live performances of the works in the portfolio. I intend to continue using modular synths and pianos and would like to explore composing more using field recordings of environmental sounds and also create soft rhythms to accompany some of these new pieces. 


\section{Bibliography}

Adelt, Ulrich. Krautrock: German Music in the Seventies. Arbor, Ann. University of Michigan Press, 2016, USA.

Adkins, Monty and Cummings, Simon. "Music Beyond Airports, Appraising Ambient Music." University of Huddersfield Press, 2019.

Alarcon, Ximena and "Pauline Oliveros: A shared resonance." Organised Sound. Herrema, Ron. Cambridge University Press, 2017.

Albiez, Sean \& Pattie, David. Kraftwerk Music Non-Stop. 2011.

Alpern, Adam. "Techniques for algorithmic composition of music."

http://hamp. hampshire. edu/adaF92/algocomp/algocomp 95, 1995.

Ančić, Ivana. "Ambijentalna Muzika Kao Praksa Popularne Umetnosti / Ambient Music as Popular Art Practice." AM: Art + Media 11, 2016.

Auner, Joseph. "Music in the Twentieth and Twenty-First Centuries." Western Music in Context. W.W. Norton \& Co, Inc. 2013.

Bates , Eliot. "Ambient Music" The SAGE International Encyclopaedia of Music and Culture. Sage Publications, 2019.

Biswas, Dipayan \& Kaisa Lund, \& Courtney Szocs "Sounds Like a Healthy Retail Atmospheric Strategy: Effects of Ambient Music and Background Noise on Food Sales." Journal of the Academy of Marketing Science, 2019

Blake, David K. "Timbre as Differentiation in Indie Music," Music Theory Online. Proquest. Chicago Vol 18, 2012.

Bohme, Gernot. "Atmosphere as a Fundamental Concept of a New Aesthetics," Thesis. 1993.

Bolanos, Gabriel. Intro to Spectralism. YouTube 2020

Boon, Marcus. "The Eternal Drone; Good Vibrations, Ancient to Future from Undercurrents," The Hidden Wiring of Modern Music. The Wire \& Rob Young 200212-15.

Bush, John. All Music Review https://www.allmusic.com/album/the-orbs-adventuresbeyond-the-ultraworld-mw0000675264 
Carr, Dan. https://reverbmachine.com/blog/deconstructing-brian-eno-music-forairports/

Carey, Liam. "Spectralism. A Short Introduction to Spectral Music." YouTube 2020

Cope, David. New Directions in Music, Fifth Edition. C M Brown Publishers, 1971

Cummings, Simon. "The Steady State Theory. Recalibrating the Quiddity of Ambient Music," 2019.

Davies, Hugh. "Dorothy Oram." Sonic Arts Network 2002.

Demers, Joanna. Listening through the Noise: The Aesthetics of Experimental Electronic Music. Published to Oxford Scholarship Online. 2010

Ellwood, David W. Rebuilding Europe: Western Europe, America and Postwar Reconstruction. London;: Longman, 1992

Eno, Brian. "Composers as Gardeners." Edge.org 2011

Eno, Brian. Generative Music A Year with Swollen Appendages. Faber \& Faber, 1996

Fox, Christopher "Editorial: Fragility and Resistance." Tempo, Cambridge University Press 2017

Frere-Jones, Sasha. "Looped In. William Basinski's evocative tape art." The New Yorker, Nov 2014.

Grant, Stuart. "Performing an Aesthetics of Atmospheres," Aesthetics 23 (1) June 2013.

Hasse, Jurgen. "Emotions in the Urban Environment: Embellishing the Cities from the Perspective of the Humanities," in Cities and Fascination, ed. Heiko Schmid, Wolf-Dietrich Sahr, and John Urry. Burlington VT: Ashgate, 2011.

Haver Currin, Grayson. "Max Richter Sleep", album review. Pitchfork Magazine, 2015.

Hegarty, Paul. Noise/Music: A History. Continuum International Publishing, 2007.

Kidd, James. "The Joys of Ambient Music. Arts and Culture," thenationalnews.com, 2018 
Kim-Cohen, Seth “Against Ambience." Essay. Music Beyond Airports. 2013.

Kramer, Jonathan D. The Time of Music. New Meanings New Temporalities New Listening Strategies. Shirmer Books, 1988.

Levaux, Christophe. "The Forgotten History of Repetitive Audio Technologies." Organised Sound; Cambridge, 2017.

Maurer iv, John. A Brief History of Algorithmic Composition. https://ccrma.stanford.edu/ blackrse/algorithm.html, 1999

McLeish, Kenneth "Aleatory (or Aleatoric)." In Bloomsbury Guide to Human Thought, edited by Bloomsbury, http://helicon.vuw.ac.nz, 1993.

Morgan, Frances. "Pioneer Spirits: New Media Representations of Women in Electronic Music History." Organised Sound: an international journal of music technology, 2017.

Murail, Tristan. "Spectra and Pixies." Contemporary Music Review, 2009.

Music History." Organised sound: an international journal of music technology 22.2 (2017)

Bruno Nettl. Nettl's Elephant: On the History of Ethnomusicology. Baltimore: University of Illinois Press, 2010.

North Christine and Dack, John. In Search of a Concrete Music, trans. (Berkeley, CA: University of California Press, 2012.

Oliveros, Pauline. Deep Listening, A Composer's Sound Practice. Deep Listening Publications, 2005.

Oram, Daphne. An Individual Note of Music, Sound and Electronics. Anomie Publishing, 2016.

Pareles, Jon. "Brian Eno's 15 Essential Ambient Works. Listen to atmospheric soundtracks for uneasy times." Playlist, The New York Times, 2020.

Prendergast, Mark. The Ambient Century. Bloomsbury Publishing, London, 2000

Reynolds, Simon. "Breaking Through the Synth Barrier. Female Artists with a Penchant for Synth Sounds." New York Times, 2011. 
Reznikoff, Legor. "Sound Resonance in Prehistoric Times: A Study of Palaeolithic Painted Caves and Rocks." The Journal of the Acoustical Society of America 123.5, 2008

Richardson, Mark. "William Basinski. The Disintegration Loops.” Pitchfork Magazine. 2012.

Rodgers, Tara. "Pink Noises: Women on Electronic Music and Sound." Duke University Press, 2010.

Rose, François Introduction to the Pitch Organization of French Spectral Music Source: Perspectives of New Music, Summer, 1996, Vol. 34, No. 2 (Summer, 1996)

Rovner, Lisa. "Sisters with Transistors. "docsinorbit.com 2021

Shepherd, Roger. In Love With These Times: My Life With Flying Nun Records. Harper Collins, 2016.

Axel Schildt et al. Between Marx and Coca-Cola: Youth Cultures in Changing European Societies, 1960-1980. 1st ed. New York, NY: Berghahn Books, 2005.

Scoates, Christopher. Brian Eno Visual Music. First Chronicle Books, 2019.

Schwarz, K. Robert. Minimalists. Phaidon Press Ltd, London, 1996.

Scheffer, Frank, and Brian Eno. Brian Eno, Music for airports: ambient music. (Place of publication not identified) Allegri Films, 1999.

Service, Tom. "Pauline Oliveros," A Guide to Contemporary Classical Music. The Guardian 2012. https://www.theguardian.com/music/tomserviceblog/2012/may/07/guidecontemporary-music-pauline-oliveros

Shanken, Edward A. Systems. Documents of Contemporary Art. White Chapel Gallery and MIT Press, 2015.

Sherburne, Philip. "About Ambient Music. The Unceasingly Curious Composer on Chance, Minimalism and the Politics of Form." Pitchfork.com, 2017.

Simoni, Mary. "Algorithmic Composition: A Guide to Composing Music with Nyquist." University of Michigan Press, 2013. 
Stubbs, David. Future Days. Krautrock and the Building of Modern Germany. Faber \& Faber, United Kingdom, 2018.

Stubbs, David. Mars By 1980. The Story of Electronic Music. Faber and Faber Ltd, Bloomsbury House, 2018.

Sherburne Philip. "A Conversation with Brian Eno About Ambient Music.” Pitchfork Magazine, 2020.

Szabo, Victor. "Unsettling Brian Eno's Music for Airports.” Twentieth-Century Music. Cambridge University Press, 2017.

Tamm, Eric. Brian Eno. His Music and Vertical Colour of Sound. Faber and Faber, 1989.

Toop, David. Ocean of Sound. Profile Books Ltd, London, 1995.

Tote, Matthew E. "La Monte Young, Terry Riley, Pauline Oliveros, and the Emergence of the American Postmodern Drone Aesthetic, 1957-1964." Tufts University, 2010.

Whitman, Keith Fullerton. The Best Ambient Albums of All Time Pitchfork.com Sep (2016)

Yueng, Vivian. "7 Essential Japanese Ambient and New Age Records." Crack Magazine. 2018. https://reverbmachine.com/blog/deconstructing-brian-eno-musicfor-airports/

Johncage.org

Krautrock - The Rebirth of Germany. BBC4 2009.

https://www.youtube.com/watch?v=QP5dOKTB3ng

http://www.coldspring.co.uk/discography/csr34cd.php

"27 essential ambient production tips. Tools and techniques for creating atmospheric sounds." Computer Music July, 2008.

Peterson, Macauley. Marcel Duchamp and John Cage "Reunion" ChessBase News, 2018

https://en.chessbase.com/post/50th-anniversary-of-reunion-and-the-death-of-marcelduchamp 
Understanding Atmosphere in Music, and How to Create it. Learn_Music And Audio Production iZotope Tips and Tutorials iSotope.com May (2018)

https://www.izotope.com/en/learn/understanding-atmosphere-in-music-and-how-to-createit.html

Frere-Jones, Sasha. Looped In. William Basinski's evocative tape art. Pop Music The New Yorker. 2014. https://www.newyorker.com/magazine/2014/11/10/looped

https://www.oxfordmusiconline.com/grovemusic/view/10.1093/gmo/978156159263 0.001.0001/omo-9781561592630-e-0000043820

https://www.researchcatalogue.net/view/393093/423723

http://www.saintmarierecords.com/products/574909-orange-the-complete-recordings

https://www.merriam-webster.com/dictionary/ambient

https://en.wikipedia.org/wiki/Ambient_music 